\title{
DETERMINACIÓN DE LOS FACTORES QUE INFLUYEN EN LA ADHERENCIA AL TRATAMIENTO EN LA DIABETES MELLITUS TIPO 2, HOSPITAL LUIS VERNAZA, PERIODO 2014-2015
}

\author{
DETERMINATION OF FACTORS THAT INFLUENCE TREATMENT ADHERENCE IN \\ TYPE 2 DIABETES MELLITUS, HOSPITAL LUIS VERNAZA, FROM 2014-2015
}

\author{
DETERMINAÇ̃̃O DOS FATORES QUE INFLUENCIAM A ADERÊNCIA AO TRATAMENTO EM \\ DIABETES MELLITUS TIPO2, HOSPITAL LUIS VERNAZA, PERÍODO 2014-2015
}

\author{
MARILYN AROSEMENA CORONEL, JULIZZA SÁNCHEZ ARMIJOS, DANIEL TETTAMANTI MIRANDA, DIEGO VÁSQUUEZ CEDEÑo, \\ MANUEL NAVARRO CHÁVEZ \\ Universidad Católica de Santiago de Guayaquil; Guayaquil, Ecuador
}

Resumen

Introducción: Los pacientes con diabetes mellitus tipo 2 de la consulta externa de endocrinología del hospital Luis Vernaza de Guayaquil, tienen dificultad para ser adherentes al tratamiento. Objetivo: determinar el porcentaje de pacientes no adherentes y cuáles son los factores más influyentes en la no adherencia al tratamiento farmacéutico y no farmacéutico. Metodología: siguiendo la Declaración de Helsinki, se presenta un estudio epidemiológico, descriptivo, analítico, observacional de corte transversal de 209 diabéticos tipo 2, realizado durante 6 meses, en la consulta externa de Endocrinología del hospital Luis Vernaza. Los pacientes llenaron un cuestionario sobre el control de diabetes y la calidad de vida. Se registraron las características demográficas y clínicas cuantitativas mediante estadística descriptiva básica y las variables cualitativas, mediante frecuencias relativas y porcentajes. La adherencia al tratamiento se consideró positiva si los pacientes cumplían cuatro criterios: ejercicio, dieta, conocimiento sobre la enfermedad y no olvidar de tomar la medicación. La ausencia de uno 0 más de esos criterios calificaba como no adherente. Resultados: de los 209 pacientes diabéticos, hubo $81 \%$ no adherentes y 19\% adherentes al tratamiento que recibían. Los factores determinantes de la no adherencia al tratamiento: no hacer dieta ( $\mathrm{p}: 0,002)$, olvidar tomar el medicamento (p:0,004) y recibir antidiabéticos orales ( $\mathrm{p}: 0.004$ ). La dieta $\mathrm{OR}>20$, olvidar de tomar la medicación $\mathrm{OR}<0.01$ y tomar antidiabéticos orales $\mathrm{OR} 0.648$. Conclusión: las variables determinantes para la no adherencia al tratamiento en los diabéticos son: no hacer dieta, olvidar tomar medicación y tener tratamiento con antidiabéticos orales. La adherencia en los pacientes atendidos en el hospital Luis Vernaza es muy baja.

PALABRAS CLAVE: diabetes mellitus tipo 2, índice glucémico, estilo de vida.

Abstract

Introduction: patients with type 2 diabetes mellitus from the endocrinology outpatient clinic of Luis Vernaza Hospital in Guayaquil have difficulty being adherent to treatment. Objective: determine the percentage of non-adherent patients and what are the most influential factors in non-adherence to pharmaceutical and non-pharmaceutical treatment. Methodology: following the Declaration of Helsinki, an epidemiological, descriptive, analytical, observational, cross-sectional study of 209 types 2 diabetics patients is presented, performed for 6 months, in the Endocrinology outpatient clinic of the Luis Vernaza Hospital. Patients filled out a questionnaire on diabetes control and life quality. Demographic and clinical quantitative characteristics were recorded using basic descriptive statistics and qualitative variables, relative frequencies, and percentages. Adherence to treatment was considered positive if the patients met four criteria: exercise, diet, knowledge about the disease, and not forgetting to take the medication. The absence of one or more of these criteria qualified as non-adherent. Results: from a total of 209 diabetic patients, there was $81 \%$ non-adherent and $19 \%$ adherent to the treatment they received. The determining factors of non-adherence treatment: no diet ( $\mathrm{p}: 0.002$ ), forgetting to take the medication ( $\mathrm{p}: 0.004)$ and receiving oral antidiabetics $(\mathrm{p}: 0.004)$. Diet $\mathrm{OR}>20$, forget to take medication $0 \mathrm{R}<0.01$, and take oral antidiabetics $\mathrm{OR} 0.648$. Conclusion: the determining variables for non-adherence treatment in diabetics are no diet, forget to take medication, and having treatment with oral antidiabetics. The adherence in the patients treated at the Luis Vernaza hospital is very low.

KEYWORDS: diabetes mellitus type 2, glycemic index, life style.

Resumo

Introdução: pacientes com diabetes mellitus tipo 2 do ambulatório de endocrinologia do hospital Luis Vernaza, em Guayaquil, têm dificuldade em aderir ao tratamento. Objetivo: determinar a porcentagem de pacientes não aderentes e quais são os fatores mais influentes na não adesão ao tratamento farmacêutico e não farmacêutico. Metodologia: após a Declaração de Helsinque, é apresentado um estudo epidemiológico, descritivo, analítico e observacional de 209 diabéticos tipo 2, realizado durante 6 meses, no ambulatório de Endocrinologia do hospital Luis Vernaza. Os pacientes preencheram um questionário sobre controle do diabetes e qualidade de vida. As características quantitativas demográficas e clínicas foram registradas usando estatística descritiva básica e variáveis qualitativas usando frequências relativas e porcentagens. A adesão ao tratamento foi considerada positiva se os pacientes atenderem a quatro critérios: exercício, dieta, conhecimento sobre a doença e não esquecer de tomar o medicamento. A ausência de um ou mais desses critérios qualificados como não aderentes. Resultados: dos 209 pacientes diabéticos, $81 \%$ não aderiram e 19\% aderiram ao tratamento que receberam. Os fatores determinantes da não adesão ao tratamento: não fazer dieta ( $p: 0,002$ ), esquecer de tomar o medicamento ( $p: 0,004)$ e receber antidiabéticos orais ( $p: 0,004)$. Dieta $0 R>20$, esqueça de tomar medicação $\mathrm{OR}<0,01$ e antidiabéticos orais $\mathrm{OR} 0,648$. Conclusão: as variáveis determinantes para a não adesão ao tratamento em diabéticos são: não fazer dieta, deixar de tomar medicamentos e fazer tratamento com antidiabéticos orais. A adesão dos pacientes atendidos no hospital Luis Vernaza é muito baixa. PALAVRAS-CHAVE: diabetes mellitus tipo 2, índice glicêmico, estilo de vida. 
INTRODUCCIÓN

La diabetes mellitus tipo 2 (DM2) es definida por la Organización Mundial de la Salud $(\mathrm{OMS})^{1}$ como una enfermedad crónica y metabólica, que aparece cuando el páncreas no produce una cantidad suficiente de insulina o cuando ésta no es utilizada eficazmente por el organismo. La insulina es la hormona que regula los niveles de glicemia y los mantiene dentro de los rangos no lesivos. ${ }^{1-5}$

La evidencia de estudios realizados en los últimos años como Ganiyu et al $^{6}$ demuestran que existe una gran proporción de diabéticos que tienen dificultad para manejar adecuadamente la medicación y mantener un estilo de vida saludable. Se definen patrones de conducta relacionados con capacidad de elegir y a las circunstancias socioeconómicas del paciente; estas circunstancias incluyen: dieta, actividad física, consumo de alcohol y tabaco. La adherencia, según lo definela $\mathrm{OMS}^{13}$; es el gradodel comportamiento de una persona: tomar el medicamento, seguir un régimen alimentario y ejecutar cambios del modo de vida, se corresponde con las recomendaciones acordadas por un prestador de asistencia sanitaria. , $^{2-25}$

Se conoce que detectando aquellos factores determinantes en la adhesión al tratamiento les permitirá a los médicos saber a dónde apuntar en la terapéutica delos diabéticos. El objetivo del estudio fue determinar cuáles son los factores más determinantes para la noadherencia al tratamiento dela DM2 y proponer posibles soluciones para mejorar estas falencias. ${ }^{26-31}$

METODOLOGía

Del 3 de junio 2014 al 6 de enero 2015 se realizó un estudio epidemiológico, descriptivo, analítico, observacional de corte transversal; fue realizado en la consulta externa de Endocrinología del hospital Luis Vernaza. Conducido para determinar los factores que más influyen en la no adherencia al tratamiento farmacéutico y no farmacéutico de los pacientes con DM2 mediante la medición de características demográficas, clínicas y un cuestionario sobre el control de la enfermedad y calidad de vida. La población estudiada estaba comprendida por personas de 30 años en adelante que habían sido diagnosticados de DM2 por lo menos hace un año, los cuales llenaron las encuestas sobre cómo llevaban su enfermedad y en qué situación estaba su tratamiento sea farmacéutico o no. Los pacientes con DM1, diabetes gestacional, menores de 30 años, récords incompletos, problemas de salud mental y pacientes en diálisis fueron excluidos del estudio. ${ }^{-9,12}$
Se realizó el cálculo del tamaño muestral tomando en cuenta un nivel de confianza del $95 \%$, error del $5 \%$ con un tamaño de la población (n aproximado) de 1000 pacientes y una distribución de las respuestas del $50 \%$. La muestra recomendada según la fórmula fue de 278 pacientes, con un método de muestreo no aleatorio.

De los 270 pacientes que constituían la población, fueron incluidos 209 que cumplieron con los criterios de inclusión y fueron excluidos 61 pacientes; 10 (diálisis), 21 (récords incompletos), 20 (incapaces de responder el cuestionario), 2 (DM1) y 8 (diagnóstico deDM2<1año).

El registro comprendía información demográfica: edad, género, estado civil, actividad económica, tiempo de diagnóstico de DM2, tratamiento que recibía, nivel de educación e IMC; además de la presión arterial, glicemia y hemoglobina glicosilada (HbA1c). Esto se realizó en una sola medición en el momento que acudieron a la consulta externa y no se realizó seguimiento de ninguno de los valores, tabla 1. Posteriormente cada paciente fue atendido y se lefueentregadoun cuestionario de 14 preguntas referentes al control de su enfermedad (calidad de vida y tratamiento), el cual fue respondido por ellos mismos. ${ }^{16}$

Las variables se midieron usando los siguientes criterios. La edad, es el tiempo transcurrido desdeel nacimiento hasta el momento de la consulta de endocrinología, el indicador son los años cumplidos y la escala es cuantitativa discreta tipo independiente. El género, se define como el estado genotípico condicionado genéticamente y que determina el género al que pertenece un individuo, puede ser masculino o femenino, la escala es nominal independiente. El estado civil, es la condición en que se encuentra la persona con relación al sexo opuesto según la ley, puede ser con o sin pareja y la escala es nominal independiente. El índice de masa corporal (IMC), es la relación entre el peso y la altura, se expresa en $\mathrm{kg} / \mathrm{m}^{2}$ y se considera normopeso: $18,5-25$, sobrepeso: $25.1-30$ y obesidad $>30$, se mide en una escala ordinal dependiente. La glicemia, es la cantidad de glucosa medida en mg/dL en sangre, se considera normal cuando se encuentra $<100 \mathrm{mg} / \mathrm{dl}$ en ayunas y anormal si es $>100 \mathrm{mg} / \mathrm{dl}$ en ayunas, se mide en una escala cuantitativa continua dependiente. La hemoglobina glicosilada (HbA1c), es una heteroproteína que resulta de la unión de la hemoglobina con glúcidos unidos a cadenas carbonadas y revela los niveles de glicemia durante los últimos tres meses, se considera una diabetes controlada cuando está 
$<7 \%$ y descontrolada si es $>7 \%$, se mide en escala cuantitativa discreta dependiente. La actividad económica, es la acción y efecto de ocupar u ocuparse, seutiliza como sinónimo de trabajo, oficio, empleoy actividad, puede ser remunerado o no remunerado, se lo mide en escala nominal independiente. Edad al diagnóstico, se define como los años cumplidos al momento del diagnóstico de la DM2, puede ser menos de 5 años, 5 a 10 años o más 10 años y se mide en escala ordinal independiente. El tratamiento, corresponde a la forma o los medios que se utilizan para controlar la glicemia, puede ser tratamiento 1: dieta y ejercicio; tratamiento 2: antiadiabéticos orales; tratamiento 3: isulina; tratamiento 4: dieta más antidiabéticos orales y tratamiento 5: dieta más insulina, se mide en escala nominal dependiente.

TABLA 1. CARACTERÍSTICAS SOCIODEMOGRÁFICAS DE LOS PACIENTES CON DM2

\begin{tabular}{|c|c|c|}
\hline GENERO & $\mathrm{N}=209$ & $\%$ \\
\hline Femenino & 141 & 67.46 \\
\hline Masculino & 68 & 32.54 \\
\hline \multicolumn{3}{|l|}{ NIVEL DE EDUCACIONN } \\
\hline No educación & 12 & 5.74 \\
\hline Educación básica & 131 & 62.68 \\
\hline Bachillerato & 58 & 27.75 \\
\hline Educación superior & 8 & 3.83 \\
\hline \multicolumn{3}{|l|}{ ESTADO CIVIL } \\
\hline Sin pareja & 69 & 33.01 \\
\hline Con pareja & 140 & 66.99 \\
\hline \multicolumn{3}{|l|}{ EDAD } \\
\hline $30-59$ años & 96 & 45.93 \\
\hline 60-79 años & 94 & 44.98 \\
\hline 80-99 años & 19 & 9.09 \\
\hline \multicolumn{3}{|l|}{ ACTIVIDAD ECONÓMICA } \\
\hline Remunerado & 47 & 22.49 \\
\hline No remunerado & 162 & 77.51 \\
\hline \multicolumn{3}{|l|}{ CARACTERISTICAS CLINICAS } \\
\hline \multicolumn{3}{|l|}{ IMC } \\
\hline Normopeso & 70 & 33.49 \\
\hline Sobrepeso & 88 & 42.11 \\
\hline Obesidad & 51 & 24.40 \\
\hline \multicolumn{3}{|l|}{ Presión arterial } \\
\hline Normotenso & 149 & 71.29 \\
\hline Hipertenso & 60 & 28.71 \\
\hline \multicolumn{3}{|l|}{ Glicemia } \\
\hline Normal & 17 & 8.13 \\
\hline Alta & 192 & 91.87 \\
\hline \multicolumn{3}{|l|}{ HbA1c } \\
\hline \multicolumn{3}{|l|}{$30-50$ años } \\
\hline Menor a $6,5 \%$ & 11 & 5.26 \\
\hline Mayor a $6,5 \%$ & 31 & 14.83 \\
\hline \multicolumn{3}{|l|}{$51-90$ años } \\
\hline Menor de 7\% & 51 & 24.40 \\
\hline Mayor a $7 \%$ & 116 & 55.50 \\
\hline \multicolumn{3}{|l|}{ Tiempo con diagnóstico de DM2 } \\
\hline $1-5$ años & 92 & 44.02 \\
\hline 5-10 años & 58 & 27.75 \\
\hline$>10$ años & 59 & 28.23 \\
\hline \multicolumn{3}{|l|}{ Tratamiento } \\
\hline Dieta y ejercicio & 18 & 8.61 \\
\hline Antidiabéticos orales & 104 & 49.76 \\
\hline Insulina & 66 & 31.58 \\
\hline Dieta más antidiabéticos orales & 20 & 9.57 \\
\hline Dieta más insulina & 1 & 0.48 \\
\hline
\end{tabular}

Se consideró a un paciente como adherente al tratamiento de DM2 si cumplía con los cuatro criterios más importantes y dependientes de la adherencia: ejercicio, dieta, conocimiento sobre la enfermedad y no olvidar de tomar la medicación. La falta de uno o más de esos criterios ya era considerada un paciente no adherente. ${ }^{1,6}$

Los análisis de los datos se realizaron utilizando el software STATA versión 11.2 para Windows ${ }^{\circledR} \mathrm{y}$ Excel para Mac $2011^{\circledR}$. Se realizó una estadística descriptiva básica en la cual se analizaron las variables cuantitativas como edad, IMC, glicemia, HbA1c, presión arterial y tiempo de diagnóstico con DM2 mediante promedios, desviación standard, error standard y sus respectivos intervalos de confianza al 95\%. (Tabla 2). En el caso de las variables categóricas cualitativas como género, actividad económica, nivel de educación, estado civil y las respuestas del cuestionario se obtuvieron frecuencias relativas y sus respectivos porcentajes. ${ }^{6,12}$ (Tabla 1), (Gráfico 1).

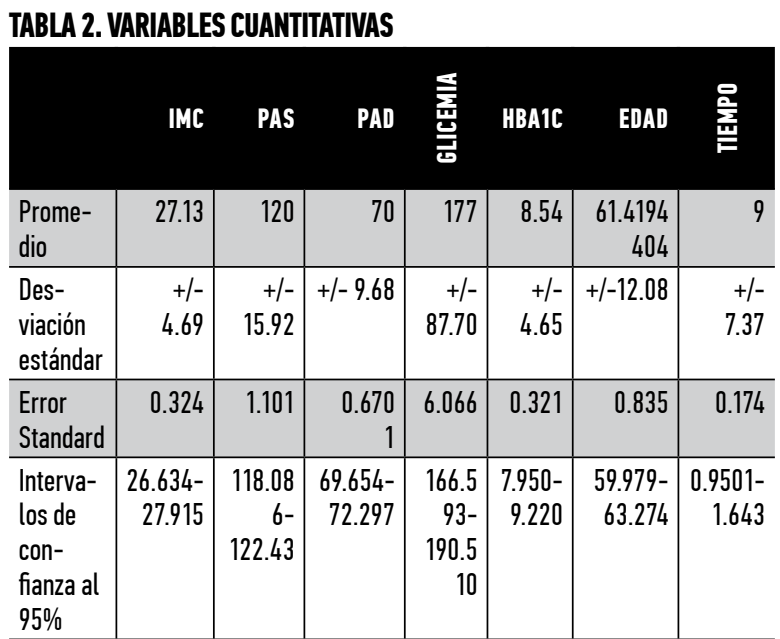

IMC: Índice de masa corporal, PAS: Presión arterial sistólica, PAD: Presión arterial diastólica, HbA1c: Hemoglobina glicosilada, TIEMPO: Tiempo con el diagnóstico de DM2.

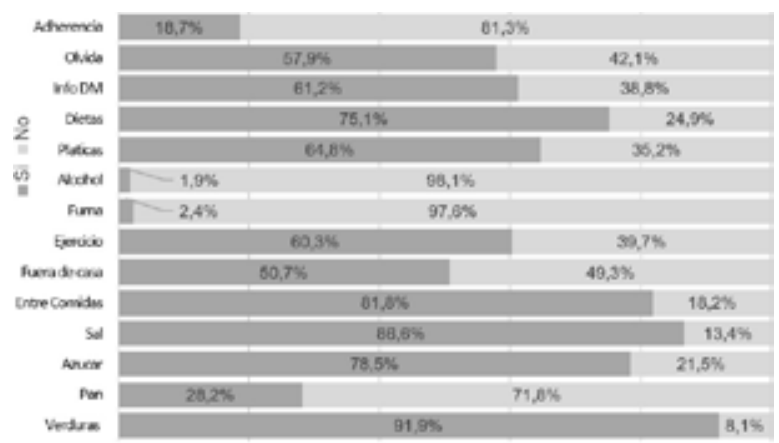

Figura 1. Respuestas en porcentajes del cuestionario de adherencia en DM2. Respuestas del cuestionario sobre adherencia al tratamiento en porcentajes. Si: Respuesta afirmativa a la pregunta $\mathrm{No}$ : Respuesta negativa a la pregunta 
Para las variables cualitativas y cuantitativas se utilizó la prueba del Chi cuadro mediante Test de Fisher; además se obtuvieron los Odds Ratio (OR) mediante regresión binaria o logística. Para las variables cualitativas ordinales se utilizó el test de Kruskal-Wallis con el cual se correlaciona este tipo de variables con la presencia o ausencia de adherencia. Además, seutilizó Chicuadradopara demostrarcuál de los parámetros determinantes de la adherencia tiene mayor significancia estadística. ${ }^{12}$

Se siguieron las recomendaciones de la Declaración de Helsinki y se obtuvo aprobación del comité científico del Hospital Luis Vernaza de la ciudad de Guayaquil previo a la realización del estudio, código: HLV-DOF-CCI-026.

RESULTADOS

Al analizar las características sociodemográficas y clínicas de los pacientes, la proporción de las mujeres y hombres era de 3:1 respectivamente, encontrando un total de 141 mujeres y 68 hombres. Con un nivel de educación básica, el 62.68\% de los pacientes; no eran remunerados, el $77.51 \%$; y tenían una pareja estable, el $66.99 \%$. La edad de la población general estaba comprendida entre 38 y 85 años, con un promedio de edad de 61.62 años y un intervalo de confianza al 95\% de 59.9-63.2. (Tabla 1)

La edad de la población femenina estaba comprendida entre 39 y 84 años, con un promedio de edad de 61.71 años; desviación estándar (DE +/-): 11.80. la edad de la población masculina estaba comprendida entre 38 y 85 años, con un promedio de edad de 61.56 años; desviación estándar (DE $+/-): 12.47$. El tiempo de diagnóstico en el género femenino tuvo un mínimo de 1 año y un máximo de 30 años; en tanto que, el género masculino tuvo un tiempo de diagnóstico mínimo de 1 año y un máximo de 32 años.

En cuanto a las características clínicas, 89 pacientes $(42 \%)$ tenían sobrepeso, con un IMC promedio de 27.28; y, 192 pacientes (92\%) mantenía una glicemia mayor a $100 \mathrm{mg} / \mathrm{dl}$ en ayunas con un promedio de $120 \mathrm{mg} / \mathrm{dl}$. La hemoglobina glicosilada (HbA1c) de los pacientes comprendidos entre 30 y 50 años se encontraba mayor a $6,5 \%$ en el $15 \%$ de lo casos; y de los pacientes comprendidos entre 51 y 90 años se encontraba mayor a 7\%, el 56\% de lo casos. Siendo $8.50 \%$ la HbA1c promedio.

En cuanto a los años de enfermedad, el 44\% tenía entre 1-5 años de diagnóstico con DM2 con un promedio de 9 años. El 28\% tenía entre 5-10 años y el $28 \%$ más de 10 años. De acuerdo al tratamiento que seguían, se encontraron 104 pacientes (50\%) recibiendo antidiabéticos orales, 66 pacientes $(32 \%)$ con insulina, 20 pacientes (10\%) haciendo dieta y recibiendo antidiabéticos orales y 18 pacientes ( $9 \%$, haciendo dieta y ejercicio. (Tabla 1). Solo 39 pacientes $(19 \%)$ mostraron una buena adherencia al tratamiento. De los cuatro parámetros seleccionados para determinar adherencia, el que tuvo menor cumplimiento fue el porcentaje de pacientes que olvidaban tomar el medicamento; 55\% para las mujeres y $65 \%$ para los hombres. ${ }^{12}$ (Tabla 3, Figuras 2 y3)

TABLA 3. FRECUENCIAS Y PORCENTAJES DE LAS VARIABLES DETERMINANTES DE LA ADHERENCIA. NFORMACIÓN SOBRE LA DM2, OLVIDA: OLVIDAR DE TOMAR LA MEDICACIÓN

\begin{tabular}{|c|c|c|c|c|}
\hline EJERCICIO & \multicolumn{2}{|c|}{ PORCENTAJE } & \multicolumn{2}{|c|}{ PORCENTAJE } \\
\hline & Mujeres & & Hombres & \\
\hline SI & 81.00 & $57.45 \%$ & 45.00 & $66,17 \%$ \\
\hline \multirow[t]{2}{*}{ NO } & 60.00 & $42.55 \%$ & 23.00 & $33,82 \%$ \\
\hline & 141.00 & $100.00 \%$ & 68.00 & $100.00 \%$ \\
\hline \multicolumn{5}{|l|}{ DIETA } \\
\hline & Mujeres & & Hombres & \\
\hline SI & 105.00 & $74.47 \%$ & 52.00 & $76.47 \%$ \\
\hline \multirow{2}{*}{ IMERDM } & 36.00 & $25.53 \%$ & 16.00 & $23.53 \%$ \\
\hline & 141.00 & $100.00 \%$ & 68.00 & $100.00 \%$ \\
\hline \multicolumn{5}{|l|}{ INFORMACIÓN } \\
\hline & Mujeres & & Hombres & \\
\hline $\mathrm{SI}$ & 103.00 & $73.05 \%$ & 50.00 & $73.53 \%$ \\
\hline NO & 38.00 & $26.95 \%$ & 18.00 & $26.47 \%$ \\
\hline & 141.00 & $100.00 \%$ & 68.00 & $100.00 \%$ \\
\hline \multicolumn{5}{|l|}{ OLVIDA } \\
\hline & Mujeres & & Hombres & \\
\hline SI se olvida & 77.00 & $54.61 \%$ & 44.00 & $64.71 \%$ \\
\hline NO se olvida & 64.00 & $45.39 \%$ & 24.00 & $35.29 \%$ \\
\hline & 141.00 & $100.00 \%$ & 68.00 & $100.00 \%$ \\
\hline
\end{tabular}

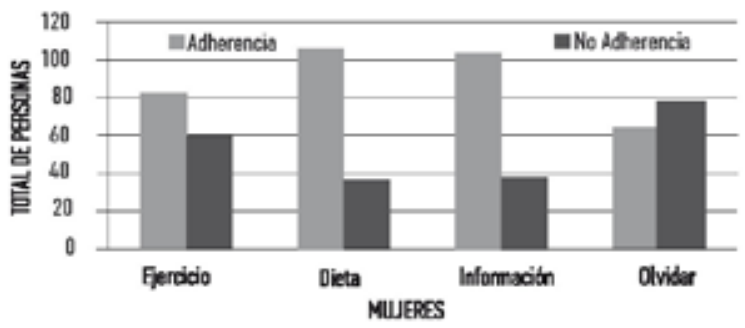

Figura 2. Frecuencias de adherencia y no adherencia en mujeres. Se observa el número de mujeres que son adherentes y no adherentes a las siguientes variables: Ejercicio, dieta, información sobre la DM2 y olvidar de tomar la medicación.

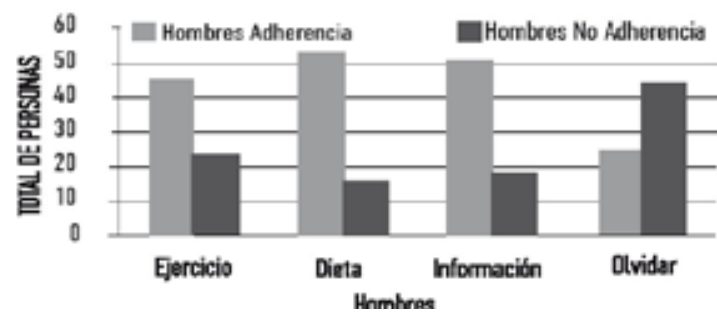

Figura 3. Frecuencias de adherencia y no adherencia en hombres. Se observa el número de hombres que son adherentes y no adherentes a las siguientes variables: Ejercicio, dieta, información sobre la DM2 y olvidar de tomar la medicación. 
Se pudo evidenciar que de las variables estudiadas, las que tenían significancia estadística $(\mathrm{p}<0,005)$ fueron: el tratamiento que recibían para la diabetes mellitus, la dieta, el ejercicio, conocer acerca de la enfermedad y no olvidarse de tomar la medicación. Siendo la más probable que aquellos pacientes que se encontraban consumiendo antidiabéticos orales (p:0.022), que no hacían ejercicio $(\mathrm{p}<0.000)$, no hacían dieta $(\mathrm{p}<0.000)$, no conocían sobre su enfermedad $(p<0.000)$ y olvidan tomar la medicación $(\mathrm{p}<0.000)$, fueran no adherentes.

DISCUSIÓN

De acuerdo a los resultados obtenidos en la regresión logística en la cual se analizaron 24 variables, se evidencia tres variables que son las más asociadas: dieta (p: 0,002), olvidar de tomar el medicamento $(\mathrm{p}: 0,004)$ y recibir antidiabéticos orales (p:0.004). Hacer dieta OR:>20 (IC al 95\%: 13.58-1179...), olvidar de tomar el medicamento OR $<0.01$ (IC al 95\% 0,001-0,000...) y tomar antidiabéticos orales OR: 0.648 (IC al $95 \%$ 0.320-1.312). Otros factores asociados son: comer fuera de la casa (p:0.034), información sobre la DM2 (0.000), comer con sal (p:0.043), fumar (p:0.000) y tomar alcohol (p:0.000). De los demás factores evaluados (género, glicemia, HbA1c, nivel de educación, etc.) a pesar que sí están asociados, no tienen una significancia estadística demostrable; por lo tanto no son relevantes.

Entre otros análisis, se evaluó la correlación entre las distintas variables. En el gráfico de cajas que correlaciona el IMC, la HbAlc y la adherencia, se observa que aquellos pacientes con un IMC mayor, tienen una mayor probabilidad de ser no adherentes al tratamiento vs aquellos con IMC más bajos, mientras que los niveles de HbA1c no varían mucho entre las dos poblaciones.

En cuanto a la correlacion entre la glicemia y la adherencia, se puede observar que hay más heterogeneidad, es incierto y no da seguridad que aquellos pacientes adherentes tengan una buena glicemia $(<100 \mathrm{mg} / \mathrm{dl})$. Lo que sí se puede afirmar es que aquellos pacientes no adherentes suelen tener glicemias alrededor de $150 \mathrm{mg} / \mathrm{dl}$ o mayores. En la distribución de edad por adherencia, se determina que el grupo adherente es el más representativo y homogéneo vs el grupo no adherente. Sin embargo no hay diferencias significativas respecto a la edad de las dos poblaciones.
A pesar de que hay más personas no adherentes con mayor antigüedad de diagnóstico de la enfermedad, no es significativo en relación a los pacientes adherentes porque tiene intervalos de confianza muy amplios, lo hace poco confiable.

La regresión logística en el análisis multivariado demostró que olvidarse de tomar la medicación, no hacer dieta y el hecho de estar en tratamiento con antidiabéticos orales, son predictores de una mala adherencia al tratamiento. Este estudio además demuestra que los pacientes con IMC mayores, tienden a ser menos adherentes. , $^{6,12}$

A diferencia de otros estudios en los que la edad es una variable significativa -y se ha demostrado que mientras la edad aumenta, aumenta la adherencia-, en este estudio no fue significativa, ya que la mayoría de los pacientes se encontraban en el mismo rango de edad. , $^{6,12}$

Otro factor que se resalta es la información sobre la enfermedad que tenían los pacientes, que demostró que aquellos pacientes que tenían menor conocimiento sobre su patología eran menos adherentes así como el ejercicio, pues aquellos pacientes que hacían menos ejercicio, eran menos adherentes. Estos resultados concuerdan por estudios previos en los que el conocimiento sobre la enfermedad juega un papel importante en la adherencia. ${ }^{6,12}$

Las modificaciones del estilo de vida son posibles en los pacientes con DM2 siempre y cuando le den importancia a las razones de una buena adherencia al tratamiento. Se debe buscar soluciones a sus necesidades y anticiparse a las futuras barreras que puedan tener. ${ }^{1-2,6,10,12}$

Por tanto el perfil de aquel paciente no adherente que debe ser identificado en la consulta desde el primer momento, es el paciente con niveles elevados de glicemia (alrededor de $150 \mathrm{mg} / \mathrm{dl}$ ), HbAlc (mayor de $7 \%$ ) e IMC (>25 kg/m2), que se alimenta fuera de su casa, ingiere alcohol y fuma; no hace dieta ni ejercicio, conoce poco sobre su enfermedad, está en tratamiento con antidiabéticos orales y olvida tomar la medicación.

De todo lo expuesto anteriormente los factores a los que hay que prestar especial atención y los cuales disminuirán las complicaciones en estos pacientes son: promover una dieta para diabético y estimular formas para que no olviden tomar la medicación. 
El presente estudio tuvo algunas limitaciones como mayor número de mujeres que de hombres, muestra significativa pero que podría ser mayor; subjetividad, al contestar ciertas preguntas sobre adherencia al tratamiento; pérdida de algunos pacientes.

\section{CONCLUSIONES Y RECOMENDACIONES}

En este estudio se determinó una asociación en la cual no hacer dieta, olvidarse de tomar la medicación y tener un tratamiento con antidiabéticos orales, hace que un paciente sea menos adherente al tratamiento. Los resultados obtenidos sobre la adherencia concuerdan con otros estudios realizados previamente en cuanto a la falta de información sobre la DM2 y el incorrecto estilo de vida que llevan los diabéticos, de manera que se podrían utilizar estrategias similares a las aplicadas en otros países. Además, la evidencia demuestra que la falta de adherencia al tratamiento es un resultado intencional de una decisión racional basada en creencias, falta de información y de autodisciplina para manejar la enfermedad. La relación médico-paciente debe enfocarse en cambiar esos paradigmas y hacer que la percepción de la DM2 sea la real con todas sus consecuencias y complicaciones.

Se debe instruir y educar a los diabéticos, eliminando falsas creencias, paradigmas o desinformación que los lleva a mantener una mala calidad de vida. Esto se basa en la promoción de salud con un correcto uso de la medicación, ejercicio diario y dieta adecuada para poder tener un buen control del metabolismo de los carbohidratos y grasas. Es necesario complementar la educación con herramientas electrónicas que tengan recordatorios para la toma de los medicamentos y adicionalmente sugieran variadas opciones de dietas, adecuadas y personalizadas según las necesidades de cada paciente. Adicionalmente, esta herramienta puede servir para que el médico acceda electrónicamente y se cerciore de que el paciente está tomando la medicación, haciendo dieta y ejercicio. De esta manera las complicaciones a corto y a largo plazo de la enfermedad disminuirán.

\section{REFERENCIAS BIBLIOGRÁFICAS}

1. Organización Mundial de la Salud, Diabetes, Nota descriptiva No. 312, Octubre 2013

2. American Diabetes Association, Standards of medical care in Diabetes, Diabetes Care Volume 37, supplement 1, 2014
3. Camarillo Guzmán, Guadalupe, Grado de apego al tratamiento en pacientes diabéticos adscritos a la Universidad de Medicina Familiar Número 66 del IMSS y sus creencias sobre el uso de terapias alternas complementarias, Instituto de Saludos Publica/UV, Universidad Veracruzana, 2012.

4. López-Carmona Juan, Construcción y validación inicial de un instrumento para medir el estilo de vida en pacientes con diabetes mellitus tipo 2, Salud Pública Méx 2003; Vol. 45(4):259-268.

5. Cramer, Joyce A., A systematic review of adherence with medications for diabetes, Diabetes Care 21:12181224, Vo 27, Number 5, 2004

6. Ganiyu Adewale B., Mabuza Langalibalele H., et al. Non-adherence to diet and exercise recommendations amongst patients with type 2 diabetes mellitus attending extension II clinic en Botswana. Afr J Prm Health Care Fam Med. 2013; 5

7. García - Pérez Luis, Álvarez María, Dilla Tatiana, et al. Adherence to therapies in patients with type 2 diabetes, Diabetes Ther 4:175-194, 2013.

8. Martínez Yolanda V, Prado-Aguilar Carlos A., et al. Quality of life associated with treatment adherence in patients with type 2 diabetes: A cross sectional study, BMC Health Services Research 2008, 8:164

9. Voorham Jaco, Haaijer-Ruskamp Flora M., Wolffenbuttel Bruce H.R, et al. Medication adherence affects treatment modifications in patients with type 2 diabetes, Clinical Therapeutics, Vo 33, number 1, 2011

10. Ho Michael P., Rumsfeld, John S., et al. Effect of medication non adherence on hospitalization and mortality among patients with diabetes mellitus, Arch Intern Med, Vol. 166, American Medical Association, 2006

11. Grant, Richard W., Singer Daniel E., et al. Polypharmacy and medication adherence in patients with type 2 diabetes, Diabetes Care, Volume 26, number 5 , American Diabetes Association 2003

12. Ahmad Nur Sufiza, Ramli Azuana, et al. Medication adherence in patients with type 2 diabetes mellitus treated at primary health clinics in Malaysia, Patient preference and adherence 2013:7 525-530.

13. World Health Organization, Defining adherence, Chapter 1, 2003

14. Blackburn D., Swidrovich J., Lemstra Mark. Non adherence in type 2 diabetes: practical considerations for interpreting the literature, Patient preference and adherence 2013 7: 183-189

15. Delamater Alan M. Improving patient adherence, Clinical diabetes, Volume 24, Number 2, 2006

16. JM López-Carmona, JR Rodríguez-Moctezuma, CR Ariza-Andraca, M Martínez-Bermúdez, "Lifestyle and metabolic control in patients with type 2 diabetes mellitus. Construct validation of IMEVID questionnaire, ”ELSEVIER Vol. 33. Núm. 01, Enero 2004. 
17. Clark, Marie. Adherence to treatment in patients with type 2 diabetes, Journal of Diabetes Nursing: Psychology series, Vol 8 No 10, 2004

18. M Millan "Quality-of-life questionnaire designed for diabetes mellitus (EsDQOL” ELSEVIER Vol. 29. Núm. 08. 15 Mayo 2002.

19. Meza Guevara Susana "Calidad de vida en pacientes ambulatorios con Diabetes Mellitus tipo 2” UANL, enero 2004.

20. Lluís Cirera, M. “Calidad de vida y salud: Diabetes Mellitus” Consejería de Sanidad, Dirección General de Salud Volumen 29. Número 700. Enero 2008.

21. Lifshitz Alberto, "El ejercicio actual de la medicina: Diabetes Mellitus" Seminario, Facultad de Medicina UNAM, 2013.

22. Grupo de trabajo de la Guía de Práctica Clínica sobre Diabetes tipo 2. Guía de Práctica Clínica sobre Diabetes tipo 2. Madrid: Plan Nacional para el SNS del MSC. Agencia de Evaluación de Tecnologías Sanitarias del País Vasco; 2008. Guías de Práctica Clínica en el SNS: OSTEBA No 2006/08

23. M. Aguilar "Criterios diagnósticos de la diabetes mellitus: Un debate permanente”Endocrinología y Nutrición, Hospital Puerta del Mar, Cádiz, JulioSeptiembre 2001.

24. 2Academia Nacional de Medicina, Buenos Aires "Guía de Práctica Clínica Nacional sobre prevención, diagnóstico y tratamiento de la Diabetes Mellitus Tipo 2" 2008
25. F.J Ampudia-Blasco et al. "Insulinización en la DM2”Unidad de Referencia de Diabetes, Servicio de Endocrinología y Nutrición. Hospital Clínico Universitario. 2008

26. Acitores A, González N, Sancho V, Valverde I, Villanueva-Peñacarrillo ML. Cell signaling of the glucagon like peptide-1 action in rat skeletal muscle. J Endocrinol. 2004; 180:389-98.

27. American Diabetes Association. Physical activity/ exercise and diabetes mellitus, 2004.

28. Grundy SM. Pre-diabetes, metabolic syndrome, and cardiovascular risk. J Am Coll Cardiol. $2012 \mathrm{Feb}$ 14;59(7):635-43

29. Gomes-Villas Boas LC, Foss MC, Freitas MC, Pace AE. Relationship among social support, treatment adherence and metabolic control of diabetes mellitus patients. Rev Lat Am Enfermagem. 2012 Feb;20(1):52-8

30. Arce, E. R. Diabetes Melitus: Programa completo para su tratamiento dietético. México: Pax México, 2005

31. Cano-Pérez, J. Guía de tratamiento de la diabetes tipo 2 en Atención Primaria. Madrid: Editorial Elsevier, 2004 